\title{
Germanica
}

\section{Innenansichten des Krieges: Histoire et autobiographie chez Dieter Wellershoff}

Innenansichten des Krieges: Geschichte und Autobiographie bei Dieter Wellershoff

Gérard Laudin

\section{CpenEdition}

Journals

Édition électronique

URL : http://journals.openedition.org/germanica/1929

DOI : $10.4000 /$ germanica. 1929

ISSN : 2107-0784

Éditeur

Université de Lille

Édition imprimée

Date de publication : 30 juin 1997

Pagination : 137-150

ISBN : 9782098426320

ISSN : 0984-2632

\section{Référence électronique}

Gérard Laudin, «Innenansichten des Krieges: Histoire et autobiographie chez Dieter Wellershoff »,

Germanica [En ligne], 20 | 1997, mis en ligne le 16 juillet 2013, consulté le 06 octobre 2020. URL : http:// journals.openedition.org/germanica/1929; DOI : https://doi.org/10.4000/germanica.1929

Ce document a été généré automatiquement le 6 octobre 2020.

(c) Tous droits réservés 


\title{
Innenansichten des Krieges: Histoire et autobiographie chez Dieter Wellershoff
}

\author{
Innenansichten des Krieges: Geschichte und Autobiographie bei Dieter \\ Wellershoff
}

Gérard Laudin

1 Depuis les années 1960, Dieter Wellershoff (né en 1925) poursuit une réflexion sur le statut littéraire du réel. Connue sous le nom de «nouveau réalisme» ou de «Kölner Schule», cette recherche phénoménologique, qui touche à la « lisibilité » du monde, au rapport de la réalité et de la fiction, du moi et du monde extérieur, soustend ses romans et s'inscrit dans les titres de ses ouvrages théoriques: Die Wahrheit der Literatur (1980), Die Arbeit des Lebens (1985), Wahrnehmung und Phantasie (1988), Der Roman und die Erfahrbarkeit der Welt (1988), Das geordnete Chaos (1992). L'essai Literatur und Lustprinzip (1973), en formulant, comme déjà Max Frisch dans les années 1960, une théorie de la littérature centrée sur le sujet, est un manifeste de la «nouvelle subjectivité » des années 70. Le réel ne pouvant être vu qu'au prisme d'un sujet qui l'interprète tout en ne le percevant que par bribes, l'écrivain doit, à la manière des nouveaux romanciers français, faire éclater la narration en perspectives subjectives, limitées, momentanées et mouvantes ${ }^{1}$, d'autant qu'il n'est plus guère en mesure de fournir comme ses devanciers du XIX ${ }^{e}$ siècle un panorama complet de la société : «Er weiß zu wenig dazu. Auch seine Erfahrungen sind zufällig und privat, und das Allgemeine liefert ihm wie allen anderen die Informationsindustrie» ${ }^{2}$.

2 Ce problème épistémologique possède un corollaire politique : c'est dans les névroses privées que s'inscrit le conflit entre l'individu et la société (ZP, 29-38). En refuser l'évocation dans les textes littéraires, c'est interdire à la littérature de remplir une fonction critique et nier, en ne tolérant que l'expression du sur-moi, la signification sociale des inadaptations personnelles. En effet: «Das Individuelle ist Abdruck des Allgemeinen, aber zugleich dessen Kritik» (ZP, 36). La littérature qui ne se défie pas du «trop privé » est ainsi «Platzhalter einer Utopie freier menschlicher Kommunikation» 
(ZP, 37) : «Ein Ich zu haben ist heute ein Potential des Widerstandes» ${ }^{3}$. Il en résulte que seul un polyperspectivisme faisant interférer des subjectivités permet d'approcher la complexité des objets. Il n'est dès lors pas étonnant que les textes littéraires de Wellershoff soient empreints d'une forte dimension autobiographique, selon des procédures proches des œuvres de Peter Handke ou Botho Strauß, ainsi qu'il apparaît dans sa première grande autobiographie, Die Arbeit des Lebens (1985)4.

Der Ernstfall. Innenansichten des Krieges (1995) applique à l'histoire du $\mathrm{III}^{\mathrm{e}}$ Reich la conception du sujet qui fonde le nouveau réalisme. CEuvre à la fois autobiographique et historienne, proche de la thématique de la "vérification d'un souvenir ${ }^{5}$, le livre repose sur une mise en perspective de l'histoire individuelle et de l'histoire collective du pays, une intention qui s'inscrit dans son projet - rendre le passé intelligible et communicable - et dans sa démarche, qui confronte le regard porté sur les événements par l'adolescent, puis le soldat des années 1935-45 avec les connaissances actuelles de portée transindividuelle, en marquant bien typographiquement les deux perspectives. L'autobiographie rétrospective réalise ainsi une ouverture vers l'histoire globale. La conception qui place au centre de l'appréhension du monde les expériences limitées du sujet a partie liée avec l'idée que l'intelligibilité de l'histoire ne saurait résulter que de la multiplication des perspectives.

4 Fondement des recherches théoriques de Wellershoff, la conception phénoménologique qui souligne la réduction extrême du regard individuel prend dans le cas des années du nazisme une dimension quasi paradigmatique, à tel point que l'expérience de la guerre peut être comprise comme la matrice du «nouveau réalisme " ${ }^{6}$. Exprimée par le soustitre, la réduction du champ visuel, évidente dans le cas de la désinformation propagandiste officielle, trouve sa forme la plus accomplie et la plus concrète dans la situation du soldat sur le champ de bataille pour qui la guerre se réduit aux quelques centaines de mètres carrés qu'il a en ligne de mire : «Das Reale war für mich nur noch der individuelle Horizont» (Spiegel-Gespräch). Comme par exemple dans le ghetto de Jakob der Lügner de Jurek Becker (1969), il s'ensuit une opposition de plus en plus forte entre l'expérience individuelle de la guerre et les événements ("das Gesamtgeschehen», «die große Lage», 275), la situation réelle du front étant largement ignorée des soldats : «Die eigene Perspektive und das Gesamtgeschehen, das Nahegelegene und das Entferntere brachen oft völlig auseinander» (102). L'expérience du front contredit ainsi un des fondements idéologiques de l'État nazi, la vision à la fois romantique et hérö̈que de la communauté : «Haupt an Haupt [...] wurde nicht gestorben, sondern jeder für sich, verteilt über ein weites Gelände, wo kaum einer den anderen sah» (82).

Der Ernstfall exemplifie ainsi les réflexions publiées en 1985 sous le titre «Das Geschichtliche und das Private. Aspekte einer Entzweiung» ${ }^{7}$. Reconstruction et non reflet du passé, le récit historique, outre qu'il transforme immanquablement le continuum historique en récit discontinu, est menacé de perdre tout sens dès lors qu'un degré excessif de conceptualisation ôte à l'histoire toute «Anschaulichkeit und Lebensnähe» (GP, 59) ou qu'inversement une reconstruction trop minutieuse des faits en dissout le sens. Il existe "eine Schwelle der Verkleinerung» (Georg Simmel), qui se confond avec une «Schwelle des historischen Interesses» (GP, 60), en deçà de laquelle le récit historique n'est plus qu'un chaos dépourvu de toute signification transindividuelle. Les cohérences de la Grande Histoire échappent au témoin direct, qui en reconstruit d'autres en fonction de son vécu propre. Il existe ainsi, parallèlement à la «Entzweiung» entre le sens privé et individuel de l'histoire et son sens collectif, une 
«erlebte Geschichte» distincte de la «erforschte Geschichte». L'écriture littéraire ou autobiographique de l'histoire trouve sa justification en ce qu'elle est susceptible, sinon de résorber, du moins de visualiser ce clivage du vécu privé et du vécu collectif (GP, 61) et de présenter, face aux travaux des historiens, un projet d'intelligiblité de l'histoire à la fois complémentaire et potentiellement opposé. Sur ce point, Wellershoff pense comme Gottfried Benn et de nombreux hommes de lettres allemands depuis Büchner8

6 La question de l'intelligibilité de la réalité historique constitue ainsi le fil conducteur de Der Ernstfall qui, en cernant quelques aspects de la psychologie de masse du fascisme, pose sans cesse la question: qu'est-ce que la réalité pour l'individu pris dans l'événement?

7 La mise en perspective des «Innenansichten» du vécu subjectif et des références objectives donne corps à des faits bien connus tels que l'absence d'informations ou la désinformation issue de la propagande officielle. Ne pouvant plus écouter la BBC, Wellershoff passe son année de formation coupé du reste du monde, «in einem Informationsvakuum [...], wie es sich heute kaum jemand vorstellen kann» (90), car même l'accès aux informations officielles est limité. A son arrivée au front en juin 1944, il ne sait rien des opérations militaires, des défaites et des pertes des six mois précédents (51-52). La pression du groupe, souvent évoquée, empêche de commenter les rares informations qui traversent les murs de la caserne. L'opacité des événements, «das Gefühl der zunehmenden Uneinheitlichkeit und Undurchschaubarkeit dieser Kriegsgesellschaft» (99), s'accroît sans cesse. Cette carence d'information entraîne la réceptivité à l'égard des rumeurs les plus étranges, comme celles concernant la mise au point d'armes nouvelles: «Da Phantasie das menschliche Vermögen ist, Informationslücken auszufüllen, und wir ständig im dunkeln tappten, waren wir anfällig für phantasieanregende Gerüchte und neue Erklärungsschemata» (96).

8 La méconnaissance du réel, voire l'inclination à reconstruire un réel imaginaire, ne touche pas les seuls civils et simples soldats. La négation de la vérité-réalité constitue un principe structurant de la pensée national-socialiste. Quand on leur livre des rapports inquiétants sur les capacités de l'industrie d'armement alliée, Göring et Goebbels réagissent selon la logique de Palmström, «daß "nicht sein kann, was nicht sein darf"» (169). Himmler consultait un astrologue dont les prédictions étaient prises très au sérieux (170), et c'est sur la base de la vision fantasmatique d'une victoire encore possible que les dirigeants nazis lancèrent fin 1944 l'attaque meurtrière des Ardennes, empêchant «ein schnelleres und für Deutschland glimpflicheres Kriegsende» (255).

9 A l'ignorance objective s'ajoute le sentiment d'irréalité de l'homme impliqué dans l'événement. Un des chapitres s'intitule de façon significative: «Ein zunehmendes Gefühl der Unwirklichkeit», tandis qu'un autre - «Freiwillig in einen verlorenen Krieg» - explique pourquoi l'auteur a pu se porter volontaire en 1943, malgré sa faible sympathie pour le régime et alors que, depuis Stalingrad, le mythe de l'invincibilité des armées allemandes commençait à s'effriter.

10 Conditionnés à l'héroïsme guerrier, élevés dans le fantasme narcissique de l'absolue supériorité des soldats allemands (49), les adolescents ressentent dans les années 1940 l'école comme un lieu à l'écart de l'histoire: «Die Reifeprüfung, so dachten viele, 
konnte man jetzt nur da bestehen, wo der Ernstfall herrschte. Dazu mußte man Soldat werden» (24). La confrontation permanente à des formes d'héroïsme nourrit des rodomontades d'adolescents mâles, mais aussi des sentiments de culpabilité et de honte quand, par exemple, un professeur de sport murmure avec mépris à chaque exercice manqué : «Armes Deutschland», faisant ainsi «aus der mißglückten Turnübung ein historisches Versagen und eine prinzipielle Unwürdigkeit» (68).

11 A cette mise en condition s'ajoutent d'autres motivations latérales telles que l'hostilité à l'égard des «Goldfasanen» et autres hommes d'appareils haïssables aux yeux de l'idéalisme juvénile (73), mais également des calculs plus pragmatiques: s'engager comme volontaire comportait en particulier l'avantage de pouvoir choisir l'unité dans laquelle on servirait, par exemple une unité où les chances de survie étaient meilleures (26-27).

Durant la formation, ces jeunes gens, à qui il manque «die ernsthafte Herausforderung durch nicht simulierte Gefechtssituationen» (84), continueront de souffrir de ce porteà-faux par rapport aux événements, de sorte qu'ils seront impatients de partir au front. La pression du groupe constitue également un facteur puissant d'intégration : «Es wäre in dieser Kompanie von Kriegsfreiwilligen nicht ratsam gewesen, offen zu bekennen, man verzichte gern auf den Frontdienst und rette lieber seine eigenen Knochen» (85).

13 Au sentiment d'irréalité, les jeunes gens répondent par une soif d'expérience vécue, qu'ils cherchent à satisfaire par-delà le bien et le mal. A propos de la participation (volontaire) à des pelotons d'exécution (dont les victimes sont souvent des soldats accusés de défaitisme), Wellershoff déclare: «Ich habe mich nie für ein solches Kommando gemeldet, kann aber nicht behaupten, daß ich dafür klare moralische Gründe gehabt hätte. Ich habe sogar mehrfach überlegt, ob ich mich nicht einmal melden sollte, um die gleiche Erfahrung zu machen wie die anderen und aus der Unwirklichkeit des Platzpatronenkrieges herauszutreten. Denn dies war zwar zweifellos das Hauptmotiv derer, die sich für das Kommando meldeten: Sie wollten wissen, wie es ist, auf einen Menschen zu schießen» (86-87).

14 La dilution du sens va jusqu'à la perte de tous les repères moraux et conduit à ne mesurer le prix de la vie qu'au critère d'intensité. Ce sentiment d'externalité revêt toutefois aussi des formes plus anodines. En 1975 déjà, Wellershoff qualifiait la guerre de «Zeit tiefster Selbstentfremdung und Betäubung»". Quand la bataille commence, il ne ressent «nichts als eine leichte Erregung» (145) ou traverse une zone découverte «wie ein Selbstmörder in Trance» (181). Transporté du front vers un hôpital du Sud, il voit l'Allemagne «wie ein vorbei wanderndes Bild, zu dem ich keine Verbindung hatte» (16). Une fois guéri, il retournera au front au terme d'une décision quasi absurde, car : «Im Lazarett [...] kam mir mein Leben hier zerschlissen und ausgehöhlt vor, als habe es sich unmerklich, aber unaufhaltsam von der Wahrheit entfernt» (263).

Le sentiment de perte du sens est renforcé par la conscience que seul le hasard règne dans les combats: «Das Sperrfeuer [...] war das Stampfen der geistlosen Wahrscheinlichkeit» (220). La reconnaissance de la dimension aléatoire de la vie humaine fut une des expériences de guerre les plus prégnantes de Wellershoff : «Denn ich wußte, [...] daß ich nur durch die vielfache Gunst des Zufalls noch lebte» (16). Elle engendra une approche existentialiste du monde : «Mit Camus kann man fordern, die fundamentale Absurdität des Lebens als Bedingung und Herausforderung anzunehmen» (74). De plus, cette expérience contredit la conception nazie de la guerre 
en montrant l'inanité de l'idéal héroïque : «Und beim Lotteriespiel kann man sich nicht bewähren» (24) ; un combat n'est que «Wette mit dem Zufall» (153).

L'expérience du front sape l'idéal de communauté. L'instinct de conservation individuelle, dans lequel Adorno et Horkheimer voient l'essence même du fascisme (Dialektik der Aufklärung) et que la politique nazie avait tout à la fois nourri et tenté d'insérer dans le culte de la communauté ethnique, triomphe au moment où le sens de l'entreprise guerrière progressivement s'estompe : «Das kollektive Drama war in viele Millionen individuelle Dramen zerfallen, und jeder einzelne kämpfte um sein Leben» (277). Cet instinct de conservation, qui fait partie des sentiments et idées inavouables ${ }^{10}$, engendre l'indifférence de chacun à l'égard de l'autre et coïncide ainsi avec le rétrécissement du champ de vision. Soigné en Bavière durant l'hiver 1944-45, Wellershoff ressent comme très lointain ce qui se passe au front: «Vage wunderte ich mich darüber, daß es mir vollkommen gleichgültig war, wie es mit meiner Kompanie weitergegangen war [...]. Es war wie ein Filmriß» (13-14).

Renforcé par les carences et les privations, l'instinct de conservation a engendré une attitude de valorisation extrême des objets matériels qui trouva après la guerre, alors que la recherche effrénée de la consommation masquait mal une perte générale du sens ${ }^{11}$, son expression littéraire par exemple dans «die Kahlschlaglyrik» de Günter Eich : «Auf dem Schlachtfeld der kollektiven Wahnideen erschienen sie als das einzig Reale. Bei Wolf gang Borchert und Heimich Boll finden sich ähnliche Einstellungen und Stimmungen bis hin zur Verklärung der einfachen Lebensnotwendigkeiten» (247). Cette attitude prolonge l'expérience de la guerre: «Dieses Vegetieren auf dem freien Feld war der Maßstab des Lebens geworden» (286).

Plus que la propagande, l'isolement individuel du « chacun dans sa nuit » et l'absence de possibilité de communiquer ôtent toute possibilité de structurer une pensée de résistance, d'autant plus que manque cruellement l'habitude d'argumenter d'un point de vue critique. Wellershoff ne se souvient pas que ses parents aient jamais tenu de propos antisémite, mais pas non plus de propos critique contre le régime: «Vorsicht wird im Spiel gewesen sein. Und vermutlich auch die Tatsache, daß sie nicht gewohnt waren, kritisch zu argumentieren»(69). La grande majorité des Allemands demeure dans le vague, l'impression ou la connaissance diffuse, l'approximatif, l'informulé, voire l'impensé : «jeder [trug] verschwiegene, meist unklare Vorbehalte in sich» (90); à leurs yeux, l'antisémitisme fait partie des «Unsäglichkeiten des Lebens» (69). Quand Wellershoff décidera de s'engager en 1943, il évitera «die Waffen-SS, die von einer Aura finsterer Gerüchte umgeben war» (27). Le vague et les effets du conditionnement idéologique s'expriment dans le «rapport contradictoire et problématique » qu'il entretenait avec la personne d'Hitler : «Um das mindeste zu sagen: Er blieb mir fremd. [...] Vermutlich hatte ich rassistische Vorurteile gegen ihn, denn meine deutsche Lichtgestalt sah aus wie der Bamberger Reiter, der in allen Schulbüchern abgebildet war» (65). Mais ces réticences sont des «unerlaubte Empfindungen» qu'il doit taire (66), de sorte qu'il s'enivre comme tant d'autres du spectacle des succès du Reich, lors des olympiades de Berlin, puis durant les premières années de guerre.

Les égarements allemands résultent d'un ensemble de causes d'importances variables : un conditionnement idéologique polymorphe, un certain nationalisme corroboré par l'absence de contre-information, l'isolement individuel, une forme d'inertie intellectuelle, l'absence de désir véritable de savoir, un conformisme imposant de faire ce que tout le monde fait, une soif juvénile d'expérience oublieuse de l'éthique et un 
certain pragmatisme. Der Ernstfall montre l'érosion progressive des positions de départ sous l'effet des dangers pesant sur la personne, jusqu'à la dissolution du «sur-moi idéologique », quand il ne reste plus que la recherche de la survie: «Als der Krieg begann, habe ich mir gewünscht, er solle so lange dauern, daß auch ich noch Soldat werden könne. Ich wollte diese Erfahrung auf keinen Fall versäumen» (148); mais une fois engagé dans l'action, tout ceci s'estompe au contact de la réalité : «[Ich] versuchte, mich zu wappnen, um mir selbst, einem alten Bild von mir selbst, treu zu bleiben. Ich will meine Sache gut machen. Man soll sich auf mich verlassen können. Aber die Dinge haben sich in letzter Zeit in mir verschoben. Hinter dem alten Vorsatz hat sich ein anderer festgesetzt, der nun meine geheime Hauptsache ist: Vor allem will ich den Krieg überleben. Möglicherweise denken das jetzt alle» (149).

Der Ernstfall constitue également une contribution à la compréhension des événements de la fin de la guerre. La propagande, dont les effets sont largement relayés par la perte $\mathrm{du}$ sens du réel, ne suffit pas à expliquer la relative cohésion de la population allemande après 1943, étant donné que Goebbels et Göring étaient depuis longtemps déconsidérés et qu'Hitler ne devait guère qu'à la rareté de ses apparitions publiques d'être relativement à l'abri des sarcasmes. La cohésion de la population tient d'abord au chaos qui règne partout, y compris dans les armées : les dignitaires du régime refusent de croire les informations alarmantes concernant l'armement allié ; les soldats ignorent à peu près tout des progrès de l'Armée Rouge ; la démoralisation de l'armée est telle que nombre d'entre eux souhaitent être faits prisonniers; des bruits circulent, expression de la crainte et d'un désir, d'une alliance des Américains et des Anglais pour faire reculer l'Armée Rouge (280). Mais c'est la peur qui soude le mieux la population :

Die Verdunkelung und Geheimhaltung der eigenen Untaten, vor allem durch das mit der Todesdrohung weitgehend durchgesetzte Verschweigen, und die Solidarisierung der Bevölkerung gegen den äußeren Feind, der ihr mit den Luftangriffen ohne Zweifel schweren Schaden zufügte, waren ein propagandistisches Doppelmanöver, das erstaunlich gut funktioniert hat. Damit daraus ein geschlossenes System, ein auswegloses Geistesgefängnis wurde, mußte allerdings auch noch der Glaube verbreitet werden, daß dem deutschen Volk im Falle einer Niederlage von den Siegern unvorstellbar Schreckliches drohe, es also zum Kampf auf Leben und Tod bis zum Ende keine Alternative gebe (59).

21 Wellershoff conteste l'identification du stalinisme à l'hitlérisme, condamné sans appel. La cruauté du maréchal soviétique qui enjoignit ses soldats de se venger sur les populations allemandes n'égale pas, pour celles-ci, les «fantasmes paranoïaques d'Hitler » qui justifie en septembre 1944 la levée de tous les hommes, de tous âges et même semi-invalides en prétextant que l'ennemi s'apprête à s'engager dans «die Ausrottung des deutschen Menschen» (60).

C'est prêter à l'ennemi ses propres obsessions destructrices au moment où la « solution finale » marche à plein. Rien de commun non plus entre cet ordre soviétique et celui de mars 1945 par lequel Hitler ordonne la destruction de toutes les infrastructures allemandes, ce qui équivaut à «eine Art Todesurteil über das deutsche Volk» (61): «[er] inszenierte seinen eigenen Untergang als ein Götterdämmerungsdrama von welthistorischem Ausmaß. [...] Wie für ihn, sollte es auch für das deutsche Volk keine Alternative mehr geben» (62). Ce délire contraste étrangement avec la réalité, la fuite éperdue de Keitel en avril 1945, racontée avec un plaisir évident : «Das Oberkommando der Wehrmacht, Hitlers militärische Paladine auf der Flucht wie wir!» (276). 
Wellershoff conçut son ouvrage en 1994 lors d'une cure à Bad Reichenhall où il avait été soigné en 1944-45. Il est d'abord confronté au caractère fugace d'un souvenir qui pourtant ressurgit de son corps (11). Ce motif proustien qu'on peut juger un peu convenu sert surtout à souligner le caractère physique de l'expérience, un thème récurrent dans son œuvre. En fait, «Die Furie des Verschwindens» (295-314) est surtout une référence hégélienne décrivant la perte de sens provoquée par la séparation de l'individuel et du collectif, «daß sich das Individuelle und Subjektive vom Allgemeinen und Absoluten trennt und dadurch in Beliebigkeit und Nichtigkeit versinkt. Am Ende dieses Prozesses steht ein bedeutungsloser, sinnloser Tod, "der keinen inneren Umfang und Erfüllung hat"»(302).

Le monument aux morts de Bad Reichenhall porte témoignage de cette perte du sens induite par le nazisme et la guerre. Si, après la Première Guerre, on avait encore pu écrire : «Ihren 200 gebliebenen Heldensöhnen zum Gedächtnis die dankbare Vaterstadt 1914-1918», l'inscription de 1945 exclut toute référence à l'héroïsme et à la patrie : «Den gefallenen Söhnen der Stadt. Bad Reichenhall 1939-1945» (302). Nulle tentative de donner un semblant de sens aux massacres: «Er [der Massentod] erscheint als das factum brutum vieler Millionen vermoderter Leichen, die das Phantasma des höheren Sinns, in dessen Dienst sie Täter und Opfer des kollektiven Mordens wurden, stumm, doch immer noch weithin hörbar verklagen» (303). Wellershoff eût perçu comme obscène une héroïsation patriotico-romantique de type hölderlinien («Der Tod fürs Vaterland»), une tentative de légitimer les massacres en arguant d'une "vaste communauté de foi symbiotique» et protectrice générant un sens supérieur (303). Cette abolition du sens est la conséquence directe de l'usage effroyable fait par les nazis des idées de communauté et de Providence pour donner une profondeur métaphysique à leurs crimes: «seitdem liegen die Kriegstoten in unserem Gedächtnis als Berge aufgehäufter Sinnlosigkeit» (304). Il en est résulté qu'aucun mécanisme de légitimation n'est resté intact en Allemagne; Wellershoff explique ainsi sa réticence à l'égard des identités collectives, son intérêt corrélatif pour l'individualité dans sa spécificité, ainsi qu'une désorientation idéologique tout à fait existentialiste : «das Glück, das darin lag, die weltanschauliche Obdachlosigkeit als geschenkte Freiheit zu erleben» (316). Cette désorientation prolonge le vague des motivations qui l'avaient conduit à s'engager : «Kriegsbegeisterung [...] war das nicht, auch keine fanatische Opferbereitschaft, sondern eher eine noch fortbestehende patriotische Konvention, gegen die man, da das zu gefährlich war, auch im Gespräch unter Freunden keine Argumente entwickelt hatte. [...] Ich zog in diesen Krieg mangels einer Alternative und ohne Illusionen, aber mit einem vagen Pflichtgefühl, das im Grunde eine Solidarität gegenüber all jenen war, die es auch getan hatten, und gegenüber den vielen, die gefallen waren» (23).

Après la guerre, il faudra à Wellershoff un long apprentissage, «Die Arbeit des Lebens», un titre qui rappelle Il mestiere di vivere (1952) de Cesare Pavese composé également après le fascisme, pour structurer en une vie des «unbestimmte offene Möglichkeiten» ${ }^{12}$ sans se départir d'une attitude d'inquiétude intellectuelle issue de l'expérience de la guerre, et cette formation individuelle s'inscrit dans la métamorphose idéologique de l'Allemagne issue de la guerre, celle de l'existentialisme, du pacifisme ou du neutralisme. Der Ernstfall réintègre ainsi le vécu privé dans le général tandis que les mouvements collectifs sont reprivatisés et dotés d'une concrétude qui est l'apport de la fiction, de l'oral story ou de l'autobiographie à la compréhension de l'histoire : «Nach 
dem Zusammenbruch der Großinterpretationen [...] ist die Autobiographie mit ihrer Perspektive der jeweils individuellen Verschiedenheit des Sehens und Deutens sehr aktuell geworden. Vielleicht ist die Erzählung des eigenen Lebens die subtilste Wahrheitsquelle, die wir haben - trotz aller Täuschungsmöglichkeiten, die auch darin stecken» (Spiegel-Gespräch).

$\mathrm{Du}$ fait du caractère aberrant des événements, tout ouvrage sur la Seconde Guerre Mondiale partage avec les œuvres littéraires d'être soumis à la nécessité de crédibilisation, en particulier auprès de la génération d'après-guerre (21). L'autobiographie peut remplir cette fonction. Toutefois, si elle peut montrer le vécu dans sa plasticité immédiate, elle n'est pas simple ressouvenir, mais réélaboration du passé, car cette perte d'immédiateté atteint même ceux qui ont vécu la guerre : «Auch ich [...] sehe heute die dokumentarischen Fotos dieser Zeit mit dem schwer zu beschreibenden Gefühlsgemisch einer ungläubigen Erinnerung. [...] Man könnte meinen, sie seien Ereignisse aus einer fiktiven Welt. [...] Der Augenschein verdeckt die inneren Bilder, und die Erinnerungen an den Krieg verwandeln sich in Bücherwissen» (22). Ce n'est pas un hasard si Der Ernstfall entretient des relations complexes avec des romans de Stendhal, Flaubert et Tolstoï évoqués dans «Das Geschichtliche und das Private». Preuves de «la vérité de la littérature » en même temps que de la modernité de ces romanciers, ces interférences témoignent d'une dialectique du vécu et du littéraire, au sens où l'expérience de la guerre a orienté le regard de Wellershoff dans la lecture de ces livres. Sans le savoir, les spectateurs du théâtre dans le Berlin des années 1940 participent, comme le héros de L'Éducation sentimentale, à la scène finale d'un «Entwicklungs- und Desillusionierungsromans» (39-40). Le récit célèbre de la bataille de Waterloo dans La Chartreuse de Parme évoque les derniers temps de la guerre dans Der Ernstfall : «Aber sonst sieht Fabrizio nicht viel von der Schlacht, denn sie setzen über Wassergräben und galoppieren durch Waldstücke. [...] Das wachsende Chaos der französischen Niederlage umgibt den orientierungslosen Fabrizio. [...] Die Anarchie ist ausgebrochen, der ungehemmte Egoismus des Willens zu überleben» (GP, 62-63). De même, c'est le polyperspectivisme que Wellershoff retient du récit de la bataille d'Austerlitz dans Guerre et paix : «Tolstoi schildert aus wechselnden Perspektiven die Vorbereitung und allmähliche Entwicklung der schlacht. [...] und noch mit schwindendem Bewußtsein begreift er [Fürst Andrej] die Nichtigkeit und Sinnlosigkeit der menschlichen Kämpfe» (GP, 64-65).

Désigné sans ambiguïté comme vision personnelle de l'époque du nazisme, Der Ernstfall participe par là-même d'une épistémologie historienne qui, critique envers l'historisme qui croit faire ressurgir le passé, nous a enseigné que le désengagement apparent du sujet n'offre qu'une garantie très formelle d'impartialité. Wellershoff, qui évite les falsifications inhérentes au témoignage personnel en confrontant ce dernier à des sources extérieures, ne prétend pas d'ailleurs faire l'histoire du IIIe Reich. Les documents cités servent certes à éclairer le point de vue individuel de perspectives étrangères, mais plus encore à montrer tout ce qui sépare la vision d'un homme pris dans l'événement de la globalité historique. Bannissant le pathétique et l'émotion qu'on trouve chez Borchert, Remarque ou Boll, plus sobre même que Jünger, Wellershoff ne cherche ni à se disculper, ni à fouiller dans ce qui serait une mauvaise conscience. Son objectif est d'analyser un aveuglement individuel et collectif. S'il rend compte sans complaisance des motivations ambiguës qui furent parfois les siennes, il soutient que l'absence d'alternative réelle exclut de s'imputer une responsabilité personnelle, mais non de souffrir du passé allemand (Spiegel-Gespräch). Sans prendre explicitement 
position dans le débat de la responsabilité collective, il semble penser que l'affirmation sans précaution de la culpabilité de tous tend dangereusement à diluer celle des grands responsables. La perspective autobiographique permet avant tout d'apporter des inflexions à la thèse de l'ignorance dans laquelle les Allemands serait demeurés. Wellershoff montre une population de «Mitläufer», à la fois «Täter und Opfer», myopes du fait de leur inclusion dans l'événement et dont la conscience est comme paralysée par les effets conjugués du conditionnement idéologique, de la peur et de la paresse intellectuelle («Nichtwissen» n'exclut pas «Nichtwissenwollen», 91) : à la propagande, ils ne parviennent dès lors qu'à opposer des réserves vagues, parce qu'ils ne disposent ni des structures de pensée, ni de valeurs critiques assez fortes à opposer au régime.

\section{NOTES}

1. - Die Wahrheit der Literatur. Sieben Gespräche (1980), p. 76.

2. - In «Zu privat. Über eine Kategorie der Verdrängung», in Literatur und Veränderung (1969), réédité dans Wahrnehmung und Phantasie. Essays zur Literatur (1987), p. 33 (ZP).

3. - D. Wellershoff, «Literatur - Widerstand mit veralteten Waffen?», in: Tintenfisch 8 (1975), p. 84.

4. - Cf. les exemples cités par Torsten Bügner, Lebenssimulationen: zur Literaturtheorie und fiktionalen Praxis von Dieter Wellershoff, Wiesbaden 1993, pp. 68-76.

5. - Motif fréquent dans le roman depuis les années 1960, «Nachprüfung einer Erinnerung» est le sous-titre du roman Zwettl de Peter Hartling (1973).

6. - Spiegel-Gespräch, n¹9/1995, pp. 86-90.

7. - In Das geordnete Chaos. Essays zur Literatur, Cologne, 1992, pp. 57-85 (GP).

8. - Gottfried Benn, «Zum Thema Geschichte», in: Gesammelte Schriften, hg. von D. Wellershoff, t. 1, pp. 371-388. - cf. notre article in: G. Laudin/Edgar Mass, Représentations de l'histoire, Cologne 1993, en part. p. 24.

9. - D. Wellershoff : «Zwischenbilanz - autobiographische Notizen», in: R. Hinton Thomas (Hg.), Der Schriftsteller Dieter Wellershoff. Interpretationen und Analysen, Köln 1975, p. 163.

10. - «Weil ich unterdrücken mußte, was ich ständig dachte, aber auf keinen Fall sagen wollte: "Hoffentlich komme ich hier raus! Ich will bloß hier raus!"» (223).

11. - Cf. Chap. «Deutschland - ein Schwebezustand», in: Die Arbeit des Lebens.

12. - «Zwischenbilanz», art. cité, p. 161.

\section{RÉSUMÉS}

Der Ernstfall. Innenansichten des Krieges (1995), récit autobiographique d'une jeunesse allemande sous le $\mathrm{III}^{\mathrm{e}}$ Reich, élargit à l'histoire les thèses phénoménologiques et existentialistes du «nouveau réalisme » que Dieter Wellershoff énonça dans les années 1960-70 et s'inscrit ainsi 
dans le prolongement de son œuvre théorique et romanesque. Comme la plupart des textes de Wellershoff, Der Ernstfall allie des considérations issues du vécu privé à des savoirs référentiels dans une interprétation partiellement anticipée par les analyses de romans de Stendhal, Flaubert et Tolstoï proposées dans un écrit théorique antérieur. Der Ernstfall exemplifie le divorce, qui atteint, dans le cas du $\mathrm{III}^{\mathrm{e}}$ Reich, une dimension quasi paradigmatique, entre l'expérience individuelle et la globalité historique, entre l'histoire vécue, perçue sur un mode fragmentaire comme par une petite fenêtre, et l'histoire globale, qui demeure opaque à l'homme pris dans l'événement. Der Ernstfall réintègre ainsi le vécu privé dans le général tandis que les mouvements collectifs sont reprivatisés et dotés d'une concrétude qui est l'apport de la fiction, de l'oral story ou de l'autobiographie à la compréhension de l'histoire. Wellershoff peut ainsi mettre en évidence la dimension matricielle de l'expérience de la guerre pour lui-même et pour la RFA, en particulier la perte du sens objectif au moment où l'éclatement du drame collectif en millions de drames privés renvoya brutalement l'individu à son intériorité.

Im Ernstfall, der Autobiographie einer deutschen Jugend im Dritten Reich, finden sich die phänomenologischen und existentialistischen Thesen des sog. «Neuen Realismus», die Wellershoff in den Jahren 1960-70 formulierte, auf die Darstellung von Geschichte angewandt. Wie die meisten seiner Texte verbindet Der Ernstfall, der auch in diesem Sinne sein theoretisches und literarisches Werk fortsetzt, Erfahrungsbericht mit Geschichtsbild in einer Interpretation, die von den in einem früheren Aufsatz erschienenen Romananalysen zu Stendhal, Flaubert und Tolstoi zum Teil antizipiert wurde. Der Ernstfall veranschaulicht die «Entzweiung», die im Fall des Dritten Reichs eine beinahe paradigmatische Bedeutung annimmt, zwischen individueller Erfahrung und Gesamtgeschehen, zwischen erlebter, als kleiner Weltausschnitt wahrgenommener Geschichte und erforschter Geschichte, die für den im Geschehen befindlichen Menschen eine völlige Undurchschaubarkeit behält. Der Ernstfall bettet das individuell Erlebte in das Kollektiv ein und führt gleichzeitig die gesamtgesellschaftlichen Erscheinungen ins Private zurück. So wird Geschichte anschaulich und lebensnah, was das Charakteristische von Fiktion, oral story oder auch autobiographischen Schriften ausmacht. So kann Wellershoff das Kriegserlebnis als Matrix diverser Aspekte seines eigenen Lebens und der Nachkriegsgeschichte aufzeigen: wie etwa Sinnverlust und «weltanschauliche Obdachlosigkeit», nachdem der Verfall des kollektiven Dramas in viele Millionen individueller Dramen den Menschen auf seine Innerlichkeit zurückgeschleudert hatte.

\section{AUTEUR \\ GÉRARD LAUDIN}

Université de Rouen 